\title{
Andreas Rauch, Musikeinsatz im Französischunterricht. Eine historische Darstellung bis 1914
}

Thèse de doctorat en Didactique du français dirigée par Marcus Reinfried, soutenue le 19 septembre 2017 à l'université de Iéna. Éditions Narr Francke Attempto, 511 pages, ISBN-10 : 3823382918

PhD thesis in Didactics of French directed by Marcus Reinfried, defended on 19 September 2017 at the University of Jena. Published by Narr Francke Attempto, 511 pages, ISBN-10: 3823382918

Andreas Rauch

\section{(2) OpenEdition}

\section{Édition électronique}

URL : https://journals.openedition.org/dhfles/7151

DOI : $10.4000 /$ dhfles. 7151

ISSN : 2221-4038

\section{Éditeur}

Société Internationale pour l'Histoire du Français Langue Étrangère ou Seconde

\section{Édition imprimée}

Date de publication : 1 décembre 2019

Pagination : 568-572

ISBN : 0992-7654

ISSN : 0992-7654

Référence électronique

Andreas Rauch, «Andreas Rauch, Musikeinsatz im Französischunterricht. Eine historische Darstellung bis $1914 »$, Documents pour I'histoire du français langue étrangère ou seconde [En ligne], 62-63 | 2019, mis en ligne le 12 avril 2020, consulté le 31 mars 2023. URL : http:// journals.openedition.org/dhfles/7151; DOI : https://doi.org/10.4000/dhfles.7151

Ce document a été généré automatiquement le 31 mars 2023.

Tous droits réservés 


\section{Andreas Rauch, Musikeinsatz im Französischunterricht. Eine historische Darstellung bis $1914^{1}$}

Thèse de doctorat en Didactique du français dirigée par Marcus Reinfried, soutenue le 19 septembre 2017 à l'université de Iéna. Éditions Narr Francke Attempto, 511 pages, ISBN-10 : 3823382918

PhD thesis in Didactics of French directed by Marcus Reinfried, defended on 19 September 2017 at the University of Jena. Published by Narr Francke Attempto, 511 pages, ISBN-10: 3823382918

\section{Andreas Rauch}

1 Le rôle de l'utilisation de la musique dans les cours de français connaît une grande popularité ces dernières décennies, surtout depuis le tournant communicatif (« kommunikative Wende ») des années 1970. Mais hormis quelques rares travaux, les aspects historiques de la place de la musique dans l'enseignement des langues n'ont guère été étudiés, notamment en ce qui concerne la période antérieure à 1914 et à l'avènement du mouvement réformiste de Wilhelm Viëtor.

\section{Objet de la recherche}

2 Ce travail de thèse constitue la première monographie ayant pour sujet l'histoire de l'utilisation de la musique dans les cours de français sur la longue durée. Elle se situe à la croisée de plusieurs champs disciplinaires : didactique, littérature, linguistique ainsi que musicologie, histoire de la musique et solfège mais aussi sociologie et sciences politiques. Cette thèse est le fruit d'une recherche diachronique approfondie sur l'utilisation de la musique dans les cours de français dans le domaine germanique, surtout dans l'enseignement en Allemagne jusqu'en 1914. Sont évoquées également les répercussions sur d'autres pays européens notamment la France et l'Italie. 
3 Selon l'aphorisme du philosophe allemand Odo Marquart, « Zukunft braucht Herkunft » («L'avenir a besoin d'origine »), la compréhension du présent se nourrit d'une connaissance et d'une analyse détaillées des évolutions historiques dans leur genèse. Le travail de thèse montre que des conceptions et des formes didactiques de l'enseignement ne constituent en aucun cas des produits du XX $\mathrm{XX}^{\mathrm{e}}$ du XXI ${ }^{\mathrm{e}}$ siècle, mais sont en germe à travers plus de 500 ans d'histoire.

4 L'objectif de ce travail est triple. Premièrement, la question est de savoir quand, où, à quelles occasions et dans quelles situations spécifiques la musique a été utilisée durant les cours. Il s'agit dans ce cas-là d'une question de didactique générale. Le deuxième objectif cherche à mettre en évidence quelles chansons furent choisies et pour quelles raisons particulières. Sont pris en compte les facteurs externes : facteurs personnels (les élèves et leurs enseignants) et fonctions (politiques, économiques, culturelles) attribués à l'apprentissage du français comme langue étrangère. En troisième et dernier lieu est présenté le vaste domaine de l'utilisation de la musique et des éléments musicaux en classe de langue. Sont réunis les facteurs internes (concernant l'enseignement en tant que tel) ainsi que les facteurs externes (notamment du domaine historico-culturel). Le corpus comprend comme sources primaires, manuels scolaires, recueils de chansons, partitions musicales, manuscrits et journaux intimes - trouvées par exemple dans le cadre des recherches à la Bibliothèque nationale de France, à la Deutsche Nationalbibliothek de Leipzig ou lors d'une vente aux enchères. Ce corpus comporte également, entre autres, l'analyse d'une liasse de 146 mémoires rédigés dans le cadre de la formation des professeurs stagiaires des lycées ("Assessorenarbeiten ») et une analyse détaillée de diverses sources secondaires disponibles notamment à la Bibliothek für Bildungsgeschichtliche Forschung à Berlin.

\section{Structure}

5 La structure de ce travail reflète son orientation diachronique. Après l'introduction qui esquisse les intentions de l'auteur et un état des recherches dans le domaine (p. 13-38) suivent trois chapitres dont le deuxième intitulé «Der Musikeinsatz im Französischunterricht im Rahmen der neusprachlichen Reformbewegung " ("L'utilisation de la musique au sein du mouvement réformiste ») est le plus central et le plus volumineux.

6 Encadrant le chapitre central, le premier chapitre traite de la période qui précède le mouvement réformiste, à savoir les origines de l'utilisation d'éléments musicaux depuis l'Antiquité jusqu'au tournant des $\mathrm{XIX}^{\mathrm{e}}-\mathrm{XX}^{\mathrm{e}}$ siècles, alors que le troisième et dernier chapitre analyse la période pendant et peu de temps après le mouvement réformiste, période que l'auteur désigne comme nouvelle «Querelle des Anciens et des Modernes ».

7 Le premier chapitre intitulé «Frühe Formen des Musikeinsatzes im Französischunterricht vor der neusprachlichen Reformbewegung" («Les premières formes de l'utilisation de la musique dans les cours de français ») analyse l'utilisation de la musique en général et puis se focalise sur l'enseignement du français en détail. Sont évoqués entre autres le rôle primordial de la Réforme et les conceptions éducatives de Martin Luther. Poète et musicien, Luther composa lui-même une soixantaine d'hymnes qui furent traduits en français. Dans les familles de confession réformée, le chant des psaumes selon le psaultier huguenot était pratiqué au sein des lectures de la Bible et l'enseignement du français servait à la préparation du culte 
calviniste. Suivent des exemples de l'enseignement du français à travers les chants religieux à Strasbourg, qui constituait en quelque sorte une plaque tournante entre la France et le Saint-Empire romain germanique, et dans des zones bilingues comme Montbéliard / Mömpelgard ou dans les colonies de huguenots de la région berlinoise qui avaient été fondées après leur fuite de la France suite à la révocation de l'Édit de Nantes.

8 Le dénominateur commun de tous ces exemples est la synthèse entre enseignement linguistique, instruction religieuse et musicale mais aussi celle du chevauchement de l'enseignement du français pour germanophones et de l'enseignement de l'allemand pour francophones. Un exemple pertinent qui illustre ce courant est celui du pasteur huguenot et pédagogue Frédéric Guillaume Hauchecorne qui fit ses études au Collège français à Berlin, établit une école dans laquelle furent utilisés non seulement la Bible et des paraboles bibliques intégrant des chants religieux avec un objectif éducatif et moral mais également des manuels novateurs s'inspirant des objets réels, les realia. De ce point de vue, Hauchecorne pourrait être considéré comme le précurseur des philanthropistes comme Basedow qui fonda en 1744 à Dessau son célèbre internat qu'il nomma Philanthropin.

9 Dans le sillage des fameuses Grammaires des Dames la question du genre joue déjà un rôle prépondérant aux XVIII ${ }^{e}$ et $\mathrm{XIX}^{\mathrm{e}}$ siècles; c'est surtout le public féminin qui est visé par une approche inductive, approche qui réunit à côté des chansons plusieurs genres et types de textes comme les danses et les rondes dans lesquels des phénomènes linguistiques et grammaticaux sont proposés pour être enseignés de façon ludique selon la devise d'une œuvre du début du XIX siècle : «Belles, chantez, instruisez-vous / Sur les règles de la Grammaire / ... / On retient plus facilement / Ce qu'on apprend en s'amusant ». Sur le frontispice à sa Grammaire en vaudevilles l'auteur Simmonin nous offre une illustration représentant trois jeunes dames qui répètent des règles de grammaire en s'accompagnant de la guitare: l'une au milieu joue du luth, une deuxième se tient debout à droite, et l'écoute, penchée vers elle, enfin à gauche, une troisième lève les deux mains au rythme de la révision grammaticale.

Le deuxième chapitre qui porte sur l'utilisation de la musique au sein du mouvement réformiste constitue le cœur de cette monographie (p. 159-350).

11 La prédominance de la méthode grammaire-traduction au début du XIX ${ }^{\mathrm{e}}$ siècle repousse au second plan l'utilisation d'éléments musicaux dans les cours de français. Le fameux manifeste de Wilhelm Viëtor «L'enseignement des langues doit faire volteface!", publié en 1882 sous le pseudonyme Quousque Tandem, marque un changement de paradigme caractérisé comme "coup de trompette" dans l'enseignement des langues. Viëtor critique la méthode grammaire-traduction et favorise la méthode directe en donnant la primauté à l'oral. En même temps, il plaide pour l'enseignement systématique de la phonétique dans les collèges et les lycées. Une importance évidente est donc accordée à la musicalité de la langue et ce phénomène est accompagné par le progrès technique et l'utilisation de grammophones dans les cours de français. Cela montre que l'intégration d'éléments musicaux constitue une composante importante inhérente au mouvement réformiste, aspect qui a été négligé jusqu’à présent.

Le troisième et dernier chapitre de la thèse analyse l'évolution de deux courants au sein $\mathrm{du}$ mouvement réformiste qui mène à une nouvelle Querelle des Anciens et des Modernes, avec d'un côté les adeptes de l'utilisation de textes contrafactum («Kontrafakturen ») en français sur des mélodies allemandes connues, et de l'autre ceux qui veulent utiliser 
(en accord avec l'épistémologie de la Kulturkunde allemande de l'époque) des chansons de facture entièrement française, texte et musique, comme éléments inhérents à la culture française. Cette querelle aboutit à un compromis (" méthode mixte ») qui, outre les chansons originales françaises, accepte également en partie l'utilisation de la Kontrafaktur (contrefacture) de mélodies allemandes.

\section{Bilan}

13 Ce travail montre que l'intégration d'éléments musicaux joue un rôle important non seulement depuis les années 1970 du XXe siècle, mais que ceux-ci ont été utilisés dans l'enseignement des langues depuis fort longtemps, à savoir depuis près de cinq siècles. L'aspect novateur en est qu'il traite d'une part de l'évolution diachronique du sujet, mais qu'il propose également des analyses détaillées synchroniques des phénomènes didactiques, culturels et sociologiques de chacune des périodes visitées. Au-delà de l'évolution retracée, la lectrice et le lecteur profiteront, grâce à la diversité des sources analysées et intégrées, d'une vue d'ensemble des méthodes d'enseignement du français jusqu'en 1914, mais dans laquelle l'utilisation de la musique constitue toutefois le fil rouge.

\section{NOTES}

1. Thèse de doctorat en Didactique du français dirigée par Marcus Reinfried, soutenue le 19 septembre 2017 à l'université de Iéna. Éditions Narr Francke Attempto, 511 pages, ISBN-10 : 3823382918.

\section{AUTEUR}

\section{ANDREAS RAUCH}

Centre de langues de l'université technique de Chemnitz 